\title{
Community-Acquired Pneumonia with Negative Chest Radiography Findings: Clinical and Radiological Features
}

\author{
Hyewon Seo ${ }^{a}$ Seung-Ick Cha ${ }^{a}$ Kyung-Min Shin ${ }^{b}$ Jae-Kwang Lim ${ }^{c}$ \\ Seung-Soo Yoo ${ }^{d}$ Shin-Yup Lee ${ }^{d}$ Jaehee Lee ${ }^{a}$ Chang-Ho Kim ${ }^{a}$ \\ Jae-Yong Park ${ }^{d}$
}

${ }^{a}$ Department of Internal Medicine, School of Medicine, Kyungpook National University, Kyungpook National University Hospital, Daegu, South Korea; ${ }^{b}$ Department of Radiology, School of Medicine, Kyungpook National University, Kyungpook National University Chilgok Hospital, Daegu, South Korea; ' Department of Radiology, School of Medicine, Kyungpook National University, Kyungpook National University Hospital, Daegu, South Korea; ${ }^{\mathrm{d}}$ Department of Internal Medicine, School of Medicine, Kyungpook National University, Kyungpook National University Chilgok Hospital, Daegu, South Korea

\section{Keywords}

Aspiration pneumonia - Community-acquired infection · Computed tomography $\cdot$ Pneumonia $\cdot$ Radiography

\footnotetext{
Abstract

Background: Data regarding community-acquired pneumonia (CAP) identified on chest computed tomography (CT) but not on chest radiography (CR) are limited. Objectives: The present study aimed to investigate the clinical and radiological features of these patients. Methods: We retrospectively compared the clinical characteristics, etiological agents, treatment outcomes, and CT findings between CAP patients with negative $C R$ and positive $C T$ findings (negative $C R$ group) and those with positive $C R$ as well as CT findings (control group). Results: Of 1,925 patients, 94 patients (4.9\%) were included in the negative CR group. Negative $C R$ findings could be attributed to the location of the lesions (e.g., those located in the dependent lung) and
}

CT pattern with a low attenuation, such as ground-glass opacity (GGO). The negative CR group was characterized by a higher frequency of aspiration pneumonia, lower incidences of complicated parapneumonic effusion or empyema and pleural drainage, and lower blood levels of inflammatory markers than the control group. On CT, the negative CR group exhibited higher rates of GGO- and bronchiolitispredominant patterns and a lower rate of consolidation pattern. Despite shorter length of hospital stay in the negative CR group, 30-day and in-hospital mortalities were similar between the two groups. Conclusions: CAP patients with negative $C R$ findings are characterized by lower blood levels of inflammatory markers, a higher incidence of aspiration pneumonia, and a lower incidence of complicated parapneumonic effusion or empyema than those with positive CR findings. Chest CT scan should be considered in suspected CAP patients with a negative $C R$, especially in bedridden patients.

(c) 2019 S. Karger AG, Basel

\section{KARGER}

(c) 2019 S. Karger AG, Basel

E-Mail karger@karger.com

www.karger.com/res
Seung-Ick Cha, MD

Department of Internal Medicine, Kyungpook National University Hospital

130 Dongdeok-ro, Jung-gu

Daegu 41944 (South Korea)

E-Mail sicha@knu.ac.kr 


\section{Introduction}

The diagnosis of pneumonia is based on clinical symptoms or signs of lower respiratory tract infection and the presence of a new infiltrate on imaging studies that cannot be explained by any other cause [1]. Although chest radiography (CR) has traditionally been used as the initial imaging modality for the evaluation of pneumonia [2], its interobserver agreement for the diagnosis of pneumonia is not perfect [3]. Thus, when clinicians rely on CR for the diagnosis of pneumonia, there is a potential risk of the diagnosis being delayed or missed $[4,5]$. Delayed diagnosis and management of pneumonia may lead to significant morbidity and mortality [5]. Chest computed tomography (CT) has been reported to be superior to CR for the visualization of lung parenchymal lesions and pleural abnormalities observed in pneumonia [1, 5-8]. In addition, CT images may be helpful in suggesting specific causative agents of pneumonia and excluding noninfectious diseases although their roles are limited [9]. CT images can also detect comorbid thoracic or pulmonary conditions [1]. Given that CT provides additional benefits for the diagnosis of pneumonia and facilitates its characterization, it is likely to be helpful when pneumonia on $\mathrm{CR}$ cannot be diagnosed definitively from CR.

Several studies have demonstrated that in some patients with pneumonia, the disease can be identified on chest CT but not on CR images $[1,4,5,7,10,11]$. Another study showed that in bedridden patients with pneumonia, the diagnostic accuracy of CR is low [6]. The location of pneumonic lesions in community-acquired pneumonia (CAP) patients with negative CR findings is different from the location in patients with positive CR findings [8]. A very recent study has suggested that the pathogens, disease severity, and outcomes are similar between these two groups of patients [12]. We hypothesized that CAP patients with negative CR findings might have clinical features distinct from those with positive CR findings. To clarify their clinical features, we compared the clinical characteristics, microbiological features, treatment outcomes, and radiologic findings between CAP patients with negative CR but positive CT findings and those with positive CR as well as CT findings.

\section{Methods}

\section{Study Design}

Consecutive patients with CAP admitted to and managed in the Division of Pulmonary and Critical Care Medicine of Kyungpook National University Hospital (KNUH), a tertiary referral center, in Daegu, South Korea, between January 2011 and November 2017 were enrolled in this study. At the time of admission, the baseline characteristics of the patients were recorded in electronic medical records, and not all patients underwent the same laboratory tests. Pneumonia was diagnosed using the following criteria [13]: (1) one or more symptoms or signs consistent with pneumonia (cough, sputum, dyspnea, fever, or pleuritic chest pain), (2) a new radiographic infiltrate, and (3) the exclusion of other causes, which was determined by follow-up CT, pathology, and culture for acid-fast bacilli. Patients with hospital-acquired pneumonia [14], healthcare-associated pneumonia [14], or an active thoracic malignancy, and those taking immunosuppressants or corticosteroids ( $>15 \mathrm{mg} /$ day of prednisone for $>14$ days) were excluded.

CR images were reviewed by two chest physicians (S.-I.C. and H.S.), and the presence or absence of pneumonic lesions, including consolidation, infiltrate, or pleural effusion, was checked. In case of conflicting opinions between the two reviewers, the decision was made by discussion till a consensus was reached. The patients were classified into two groups: (1) CAP patients with negative CR but positive CT findings (negative CR group) and (2) those with positive CR as well as CT findings (control group). Clinical variables, including clinical characteristics, microbiological features, treatment outcomes, and radiologic findings, were compared between the negative CR and control groups. The study was approved by the Institutional Review Board of $\mathrm{KNUH}$, which waived the requirement for written informed patient consent because of the retrospective nature of the study.

\section{CT Findings}

Two chest radiologists (J.-K.L. and K.-M.S.) reviewed the chest CT scans of CAP patients for the presence of consolidation, ground-glass opacity (GGO), and centrilobular nodules or tree-inbud appearance. The patients were classified into three groups based on the CT pattern (Fig. 1): (1) the bronchiolitis-predominant pattern, i.e., centrilobular nodules or tree-in-bud appearance in most lesions with minimal GGO or consolidation; (2) the GGOpredominant pattern, i.e., focal or diffuse GGO with no or minimal centrilobular nodules, tree-in-bud appearance, or consolidation; and (3) the consolidation pattern, i.e., consolidation with or without variable extents of centrilobular nodules or GGO [15]. The presence of necrotizing pneumonia and the presence and extent of pleural effusion were also examined. Necrotizing pneumonia was defined as the presence of consolidation with multiple areas of low density in one or more segments or lobes on enhanced CT images [16]. The extent of pleural effusion was classified as follows: small, $<2 \mathrm{~cm}$ at maximal depth; moderate, $2-5 \mathrm{~cm}$; and large, $>5 \mathrm{~cm}$. Dependent locations of the lungs included the posterior segments of the upper lobes and the superior and posterior basal segments of the lower lobes.

\section{Data Collection}

Demographic data, including age, sex, smoking history, and alcohol consumption, were reviewed. Heavy drinking was defined as the consumption of 7 or more drinks ( $>60 \mathrm{~g}$ of alcohol) on a single occasion for men, and 5 or more drinks ( $>40 \mathrm{~g}$ of alcohol) on a single occasion for women at least twice a week. Data on symptoms, vital signs, comorbidities, pneumonia severity indices (PSI) [17], CURB-65 scores [18], and Eastern Cooperative Oncology Group (ECOG) performance status [19] were also collected, and the Charlson Comorbidity Index [20] was retrospectively cal- 

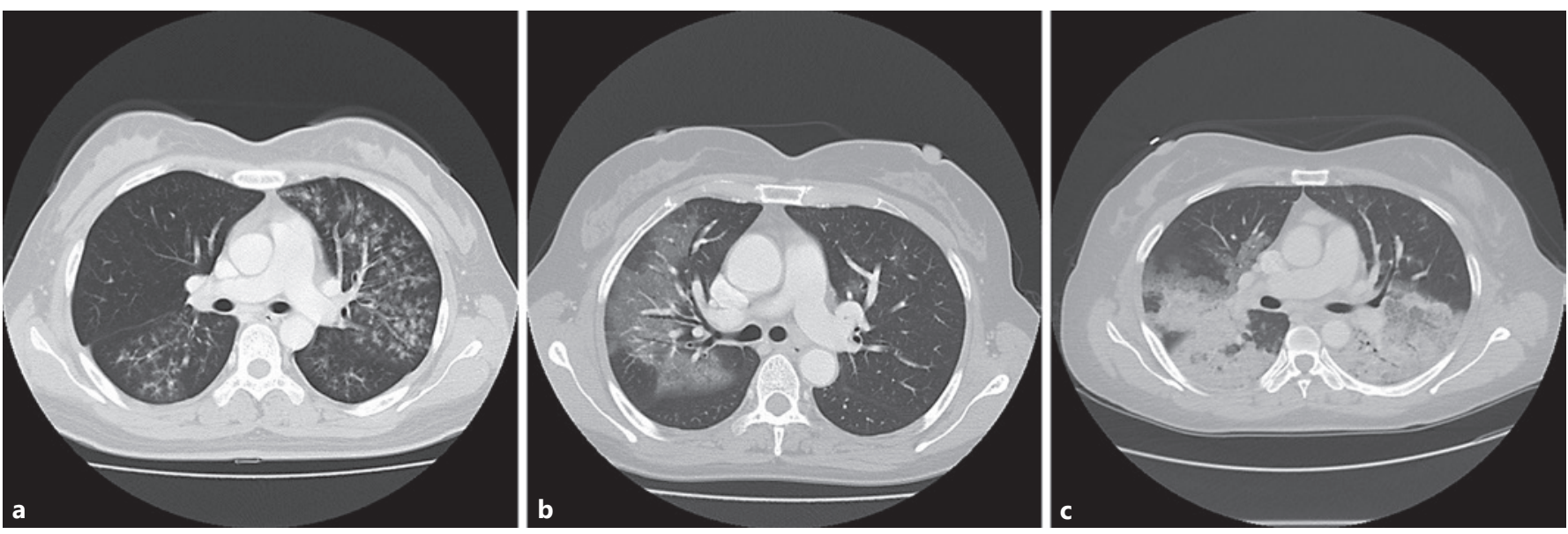

Fig. 1. Computed tomography patterns in patients with community-acquired pneumonia. a Bronchiolitis-predominant pattern, centrilobular nodules or tree-in-bud appearance in most lesions with minimal ground glass opacity (GGO) or consolidation.

b GGO-predominant pattern, focal or diffuse GGO with no or minimal centrilobular nodules, tree-in-bud appearance, or consolidation. c Consolidation pattern, consolidation with or without variable extents of centrilobular nodules or GGO.

Fig. 2. Flow chart of the study protocol. CT, computed tomography; $\mathrm{CR}$, chest radiography.

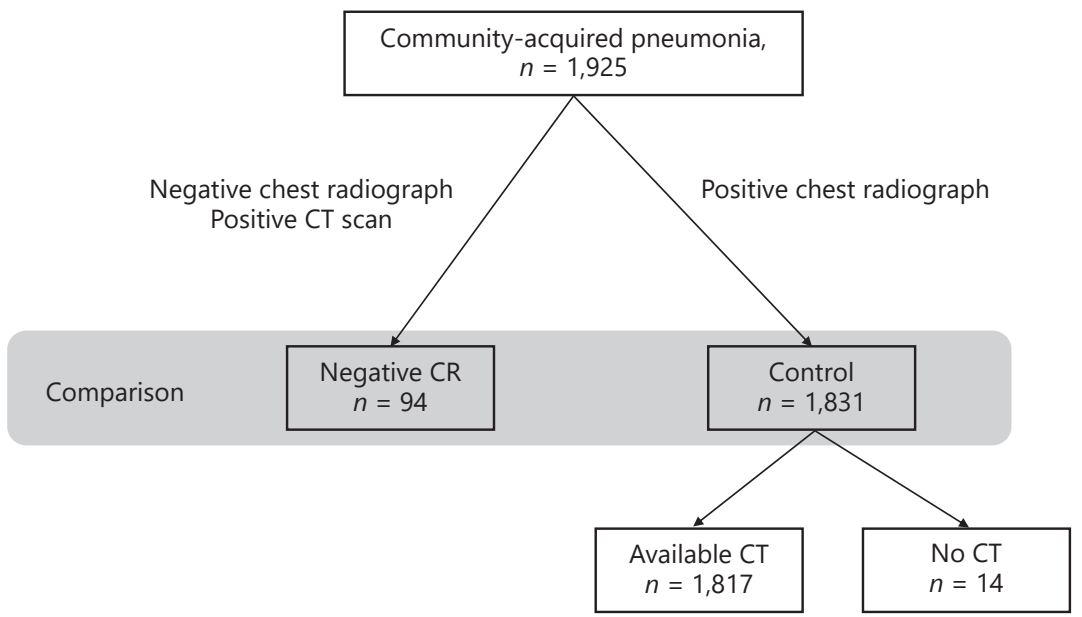

culated. Aspiration pneumonia was defined as pneumonia with radiographic evidence of infiltration in the superior or posterior basal segments of the lower lobes or posterior segments of the upper lobes in patients with a history of vomiting or witnessed aspiration or with risk of aspiration [21]. Use of mechanical ventilation, corticosteroid treatment, vasopressor infusion, and pleural drainage with percutaneous catheters or chest tubes were checked. Outcome variables included length of hospital stay (LOS), 30-day mortality, in-hospital mortality, and treatment success. Treatment success was defined as improvements in clinical symptoms and/or signs and radiologic findings. Laboratory data included complete blood count, erythrocyte sedimentation rate, C-reactive protein, procalcitonin, N-terminal prohormone brain natriuretic peptide (NT-proBNP), blood urea nitrogen, creatinine, sodium, lactate de- hydrogenase, lactate, and results of liver function tests and arterial blood gas analysis.

\section{Microbiological Data}

Causative pathogens were determined in cases in which any of the following criteria [16] were met: detection of a microorganism cultured from blood or pleural fluid; positive urinary antigen test for Streptococcus pneumoniae or Legionella pneumophila serogroup 1 (BinaxNOW ${ }^{\circledR}$ S. pneumoniae and Legionella urinary antigen cards, Alere, Scarborough, ME, USA); isolation of bacteria from sputum culture ( $>25$ neutrophils and $<10$ squamous epithelial cells per low-power field) collected within $24 \mathrm{~h}$ of admission with a compatible Gram stain finding; identification of Mycoplasma pneumoniae based on a positive immunoglobulin M (IgM) re- 


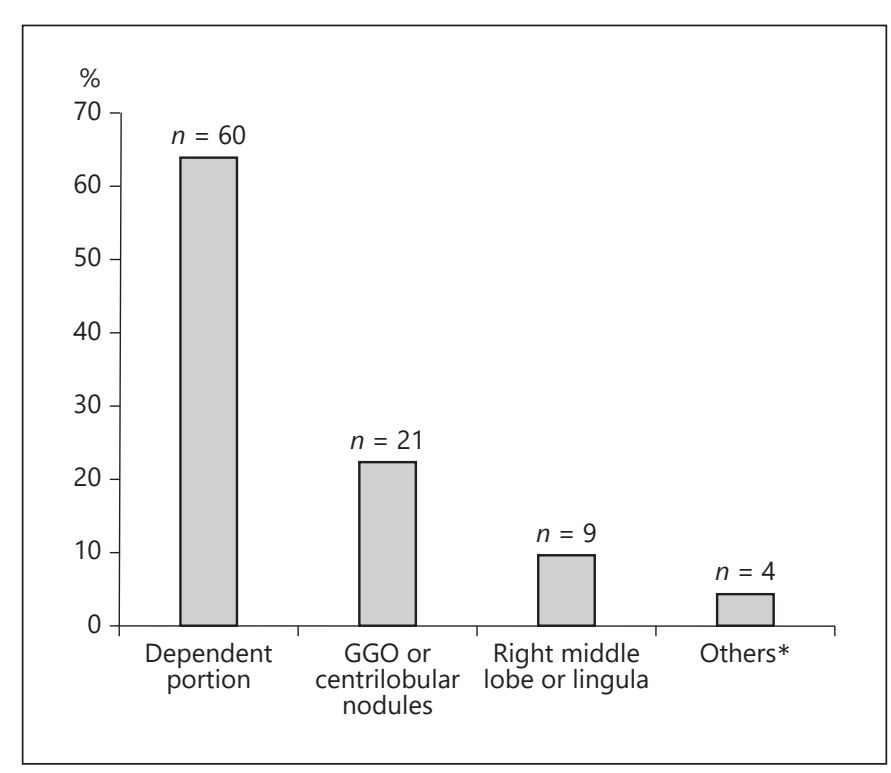

Fig. 3. Causes of negative findings on chest radiographs. The most common cause is the location of lesions in dependent regions of the lungs (60 [63.8\%]), followed by computed tomography pattern (21 [22.3\%]), including ground-glass opacity (GGO) or centrilobular nodules, and presence of the lesions in the right middle lobe or lingula (9 [9.6\%]). * Others include focal consolidation of the right upper lobe ( $n=2$, apical and anterior segments) and multifocal nodular or patchy lesions $(n=2)$.

sult in blood or a fourfold increase in immunoglobulin G (IgG) levels in convalescent versus initial blood samples by chemiluminescence immunoassay (LIAISON, DiaSorin, Saluggia, Italy) or positivity for $M$. pneumoniae in sputum by polymerase chain reaction (PCR) (AmpliSens ${ }^{\circledR}$ Mycoplasma pneumoniae-FEP PCR, Central Research Institute for Epidemiology, Moscow, Russia); identification of Chlamydia pneumoniae as determined by the presence of IgM in blood or a fourfold increase in IgG levels by microimmunofluorescence (an in-house method) or by enzymelinked immunosorbent assay (Diesse Diagnostica Senese, Monteriggioni, Italy); identification of influenza A or B antigen in a throat swab by rapid chromatographic immunoassay (SD BIOLINE Influenza Antigen test, Standard Diagnostics, Yongin, Korea or BD Veritor System for Rapid Detection of FluA+B, BD Diagnostics, Sparks, MD, USA); and positivity for a respiratory virus in a throat or nasopharyngeal swab as determined by multiplex PCR (Anyplex ${ }^{\circledR}$ RV16 detection, Seegene, Seoul, Korea).

\section{Statistical Analysis}

Data were expressed as medians with interquartile ranges for continuous variables and as numbers with percentages for categorical variables. Continuous variables were compared between groups using the Mann-Whitney $U$ test, and categorical variables were compared using the $\chi^{2}$ test. $p$ values $<0.05$ were considered statistically significant. Statistical analysis was performed using IBM SPSS Statistics for Windows, version 23.0 (IBM Corp., Armonk, NY, USA) software.

Community-Acquired Pneumonia with

Negative Chest Radiograph

\section{Results}

\section{Enrolled Patients}

Overall, 1,925 CAP patients were identified. The agreement between the two readers regarding the presence or absence of positive findings on CR was good $(\kappa=0.603)$. The patients were classified into the negative CR $(n=94[4.9 \%])$ and control $(n=1,831)$ groups (Fig. 2). The reasons for the absence of positive CR findings are presented in Figure 3. The most common cause was the location of the lesions in the dependent regions of the lung (60 [63.8\%]), followed by GGO- or bronchiolitis-predominant pattern $(21$ [22.3\%]) and presence of lesions in the right middle lobe or lingula (9 [9.6\%]). In the negative CR group, the anterior-posterior chest view was more frequently obtained (29 [30.9\%] vs. 334 [18.2\%], $p=0.002$ ) and the lateral view was less frequently obtained (57 [60.6\%] vs. $1,326[72.4 \%], p=$ 0.013 ) compared with the control group. Of the $60 \mathrm{pa}-$ tients with lesions located in the dependent lung, $25 \mathrm{pa}$ tients $(41.7 \%)$ had only the anterior-posterior chest view and 31 patients $(51.7 \%)$ did not undergo the lateral view.

\section{Clinical Characteristics and Antibiotic Therapy}

Demographic characteristics did not differ significantly between the negative CR and control groups (Table 1). The Charlson Comorbid Index was not significantly different between the two groups, but the incidences of ECOG 4 (14 [14.9\%] vs. 103 [5.6\%], $p<0.001)$ and bedridden status $(10[10.6 \%]$ vs. 66 [3.6\%], $p=0.003)$ were significantly higher in the negative CR group compared with the control group. The frequency of aspiration pneumonia was also significantly higher in the negative CR group (16 [17.0\%] vs. 109 [6.0\%], $p<0.001)$. The negative CR group had significantly lower frequencies of complicated parapneumonic effusion or empyema (2 [2.1\%] vs. $186[10.2 \%], p=0.011)$ and pleural drainage (3 [3.2\%] vs. $225[12.3 \%], p=0.008)$ than the control group. The proportions of patients with high CURB-65 scores (score 3-5) and high PSI (class 4-5) were similar between the two groups. With regard to the treatment outcome, LOS was significantly shorter in the negative CR group, whereas 30-day mortality, in-hospital mortality, and rate of treatment success were not significantly different between the two groups. Initial antibiotic therapy was similar between the two groups, except for antipseudomonal beta-lactam-based regimen (1 [1.1\%] vs. 163 [8.9\%], $p=$ 0.008 ), which was administered less frequently in the negative CR group (Table 2). 
Table 1. Baseline characteristics of the patients $(n=1,925)$

\begin{tabular}{|c|c|c|c|}
\hline Characteristics & $\begin{array}{l}\text { Control } \\
(n=1,831)\end{array}$ & $\begin{array}{l}\text { Negative CR } \\
(n=94)\end{array}$ & $p$ value \\
\hline Age, years & $69(56-77)$ & $66.5(52-76)$ & 0.208 \\
\hline Male sex & $1,243(67.9)$ & $59(62.8)$ & 0.301 \\
\hline \multicolumn{4}{|l|}{ Smoking } \\
\hline Ever-smoker & $1,044(57.2)$ & $49(52.1)$ & 0.332 \\
\hline Pack-years & $10(0-35)$ & $5(0-30)$ & 0.184 \\
\hline Heavy drinker & $325(17.7)$ & $15(16)$ & 0.657 \\
\hline Charlson Comorbidity Index & $0(0-1)$ & $0(0-1)$ & 0.277 \\
\hline ECOG & $1(1-2)$ & $1(1-2)$ & 0.900 \\
\hline ECOG 4 & $103(5.6)$ & $14(14.9)$ & $<0.001$ \\
\hline Bedridden status & $66(3.6)$ & $10(10.6)$ & 0.003 \\
\hline Systolic blood pressure, $\mathrm{mm} \mathrm{Hg}$ & $130(112-147)$ & $126(104-149)$ & 0.287 \\
\hline Pulse rate/min & $96(83-110)$ & $92(81-102)$ & 0.042 \\
\hline Respiratory rate/min & $20(19-22)$ & $20(18-20)$ & 0.003 \\
\hline \multicolumn{4}{|l|}{ Symptoms } \\
\hline Duration of symptom, days & $5(3-7)$ & $5(3-7)$ & 0.116 \\
\hline Cough & $1,599(87.3)$ & $76(80.9)$ & 0.068 \\
\hline Sputum production & $1,321(72.1)$ & $66(70.2)$ & 0.684 \\
\hline Dyspnea & $1,047(57.2)$ & $35(37.2)$ & $<0.001$ \\
\hline Fever & $1,204(65.8)$ & $58(61.7)$ & 0.420 \\
\hline Altered mental status & $84(4.6)$ & $8(8.5)$ & 0.085 \\
\hline Hemoptysis & $138(7.5)$ & $8(8.5)$ & 0.728 \\
\hline Chest pain & $456(24.9)$ & $15(16.0)$ & 0.049 \\
\hline CURB-65 score $3-5$ & $178(9.7)$ & $8(8.5)$ & 0.698 \\
\hline PSI class $4-5$ & $658(35.9)$ & $27(28.7)$ & 0.154 \\
\hline Aspiration pneumonia & $109(6.0)$ & $16(17.0)$ & $<0.001$ \\
\hline Complicated parapneumonic effusion or empyema & $186(10.2)$ & $2(2.1)$ & 0.011 \\
\hline Pleural drainage & $225(12.3)$ & $3(3.2)$ & 0.008 \\
\hline Mechanical ventilation & $109(6.0)$ & $3(3.2)$ & 0.265 \\
\hline Corticosteroids & $421(23.0)$ & $8(8.5)$ & 0.001 \\
\hline Vasopressor infusion & $163(8.9)$ & $4(4.3)$ & 0.119 \\
\hline 30-day mortality & $147(8.0)$ & $6(6.4)$ & 0.565 \\
\hline In-hospital mortality & $132(7.2)$ & $4(4.3)$ & 0.276 \\
\hline Length of hospital stay, days & $9(6-13)$ & $7(4-9)$ & $<0.001$ \\
\hline Treatment success & $1,645 / 1,793(91.7)$ & $87 / 93(93.5)$ & 0.536 \\
\hline
\end{tabular}

Data are presented as median (interquartile range) or $n$ (\%). ECOG, Eastern Cooperative Oncology Group performance status; CURB-65, a six-point score, one point for each of confusion, urea $>7 \mathrm{mmol} / \mathrm{L}$, respiratory rate $\geq 30 / \mathrm{min}$, low systolic ( $<90 \mathrm{~mm} \mathrm{Hg}$ ) or diastolic ( $\leq 60 \mathrm{~mm} \mathrm{Hg}$ ) blood pressure, and age $\geq 65$ years; PSI, pneumonia severity index.

\section{Laboratory Data and Microbiologic Results}

The laboratory data of the participants are presented in Table 3. The negative CR group had significantly lower blood levels of inflammatory markers, including erythrocyte sedimentation rate $(34 \mathrm{~mm} / \mathrm{h}[17-47 \mathrm{~mm} / \mathrm{h}]$ vs. 45 $\mathrm{mm} / \mathrm{h}[28-64 \mathrm{~mm} / \mathrm{h}], p<0.001)$, C-reactive protein $(7.0$ $\mathrm{mg} / \mathrm{dL}$ [3.3-11.8 mg/dL] vs. $13.1 \mathrm{mg} / \mathrm{dL}$ [6.7-21.2 mg/ $\mathrm{dL}], p<0.001)$, and procalcitonin $(0.12 \mathrm{ng} / \mathrm{mL}[0.05-0.55$ $\mathrm{ng} / \mathrm{mL}]$ vs. $0.35 \mathrm{ng} / \mathrm{mL}[0.10-1.64 \mathrm{ng} / \mathrm{mL}], p<0.001)$. Serum NT-proBNP level was also significantly lower (221 $\mathrm{pg} / \mathrm{mL}$ [59-953 ng/mL] vs. $432 \mathrm{ng} / \mathrm{mL}[138-1,595 \mathrm{ng} /$ $\mathrm{mL}], p<0.001$ ) in the negative CR group than the control group. The negative CR group had a significantly higher ratio of partial pressure of arterial oxygen to fraction of inspired oxygen than the control group $(372 \mathrm{~mm} \mathrm{Hg}$ [297-423 mm Hg] vs. $346 \mathrm{~mm} \mathrm{Hg}$ [280-411 mm Hg], $p=0.034)$.

Data regarding causative microorganisms are summarized in Table 4. Of the 1,925 CAP patients, potential pathogens were identified in 842 patients (43.7\%): 45/94 
Table 2. Initial antibiotic treatment

$\begin{array}{ll}\begin{array}{l}\text { Control } \\ (n=1,831)\end{array} & \begin{array}{l}\text { Negative CR } \\ (n=94)\end{array} \quad p \text { value }\end{array}$

Third-generation cephalosporin with or without macrolide or fluoroquinolone

Third-generation cephalosporin plus clindamycin

Ampicillin-sulbactam with or without macrolide or fluoroquinolone

Fluoroquinolone with or without aminoglycoside

Antipseudomonal beta-lactam with or without macrolide, fluoroquinolone, or aminoglycoside

Carbapenem with or without macrolide, fluoroquinolone, or aminoglycoside

Glycopeptide plus antipseudomonal beta-lactam with fluoroquinolone or carbapenem with or without macrolide, fluoroquinolone, or aminoglycoside

Others*

$\begin{array}{ccc}1,023(55.9) & 61(64.9) & 0.085 \\ 54(2.9) & 2(2.1) & >0.999 \\ & & \\ 453(24.7) & 25(26.6) & 0.685 \\ 64(3.5) & 4(4.3) & 0.571 \\ & & \\ 163(8.9) & 1(1.1) & 0.008 \\ & & >0.999 \\ 17(0.9) & 0(0.0) & \\ & & 0.723 \\ 44(2.4) & 1(1.1) & >0.999\end{array}$

Data are presented as $n(\%) .{ }^{*}$ Others include macrolide alone $(n=1)$, glycopeptide plus clindamycin $(n=1)$, fluoroquinolone plus aminoglycoside plus clindamycin $(n=1)$, antipseudomonal beta-lactam plus clindamycin $(n=1)$, third-generation cephalosporin plus glycopeptide $(n=1)$, carbapenem plus aminoglycoside plus macrolide $(n=1)$, fluoroquinolone plus clindamycin $(n=2)$, fluoroquinolone plus glycopeptide $(n=2)$, macrolide plus glycopeptide $(n=1)$, and third-generation cephalosporin plus aminoglycoside $(n=2)$.

Table 3. Blood laboratory findings of the patients

\begin{tabular}{|c|c|c|c|c|c|}
\hline Parameters & Control & $n$ & Negative CR & $n$ & $p$ value \\
\hline WBC count $/ \mu \mathrm{L}$ & $10,740(7,710-15,290)$ & 1,831 & $10,480(6,828-14,635)$ & 94 & 0.562 \\
\hline $\mathrm{ESR}, \mathrm{mm} / \mathrm{h}$ & $45(28-64)$ & 1,824 & $34(17-47)$ & 93 & $<0.001$ \\
\hline C-reactive protein, $\mathrm{mg} / \mathrm{dL}$ & $13.1(6.7-21.2)$ & 1,826 & $7.0(3.3-11.8)$ & 94 & $<0.001$ \\
\hline Procalcitonin, ng/mL & $0.35(0.10-1.64)$ & 1,719 & $0.12(0.05-0.55)$ & 88 & $<0.001$ \\
\hline NT-proBNP, pg/mL & $431.5(138.2-1,595.3)$ & 1,720 & $221.0(59.3-953.0)$ & 86 & $<0.001$ \\
\hline Troponin I, ng/mL & $0.015(0.015-0.029)$ & 1,518 & $0.015(0.015-0.021)$ & 73 & 0.634 \\
\hline Hemoglobin, g/dL & $12.4(11.2-13.6)$ & 1,831 & $13.3(11.9-14.5)$ & 94 & $<0.001$ \\
\hline Platelet, $10^{3} / \mu \mathrm{L}$ & $238(176-321)$ & 1,831 & $229(179-301)$ & 94 & 0.547 \\
\hline Albumin, $\mathrm{g} / \mathrm{dL}$ & $3.4(2.9-3.8)$ & 1,831 & $3.7(3.3-4.1)$ & 94 & $<0.001$ \\
\hline Total protein, g/dL & $6.4(5.9-6.9)$ & 1,831 & $6.8(6.1-7.3)$ & 94 & $<0.001$ \\
\hline Total bilirubin, $\mathrm{mg} / \mathrm{dL}$ & $0.60(0.39-0.90)$ & 1,830 & $0.58(0.40-0.89)$ & 94 & 0.786 \\
\hline $\mathrm{AST}, \mathrm{U} / \mathrm{L}$ & $25(18-39)$ & 1,830 & $25(17-33)$ & 94 & 0.329 \\
\hline $\mathrm{ALT}, \mathrm{U} / \mathrm{L}$ & $20(13-32)$ & 1,830 & $19(13-30)$ & 94 & 0.628 \\
\hline ALP, U/L & $77(62-106)$ & 1,827 & $73(59-94)$ & 94 & 0.058 \\
\hline BUN, mg/dL & $15.8(11.3-23.8)$ & 1,831 & $15.2(11.4-22.8)$ & 94 & 0.790 \\
\hline Creatinine, $\mathrm{mg} / \mathrm{dL}$ & $0.90(0.70-1.20)$ & 1,831 & $0.90(0.66-1.24)$ & 94 & 0.728 \\
\hline Sodium, mmol/L & $136(134-139)$ & 1,831 & $137(134-139)$ & 94 & 0.207 \\
\hline $\mathrm{PaO}_{2} / \mathrm{FiO}_{2}, \mathrm{~mm} \mathrm{Hg}$ & $345.5(280.1-410.5)$ & 1,624 & $372.0(296.8-423.3)$ & 76 & 0.034 \\
\hline $\mathrm{PaCO}_{2}, \mathrm{~mm} \mathrm{Hg}$ & $28.8(26.0-32.2)$ & 1,651 & $31.9(27.9-35.8)$ & 77 & $<0.001$ \\
\hline $\mathrm{LDH}, \mathrm{U} / \mathrm{L}$ & $360(256-471)$ & 1,321 & $352(319-423)$ & 59 & 0.700 \\
\hline Lactate, $\mathrm{mmol} / \mathrm{L}$ & $1.9(1.4-2.7)$ & 1,187 & $1.8(1.5-2.4)$ & 58 & 0.881 \\
\hline
\end{tabular}

Data are presented as median (interquartile range). WBC, white blood cell; ESR, erythrocyte sedimentation rate; NT-proBNP, N-terminal of prohormone brain natriuretic peptide; LDH, lactate dehydrogenase; AST, aspartate aminotransferase; ALT, alanine transaminase; ALP, alkaline phosphatase; BUN, blood urea nitrogen. 
Table 4. Microbiologic diagnosis

\begin{tabular}{|c|c|c|c|}
\hline & $\begin{array}{l}\text { Control } \\
(n=797)\end{array}$ & $\begin{array}{l}\text { Negative CR } \\
(n=45)\end{array}$ & $p$ value \\
\hline Streptococcus pneumoniae & $183(19.2)$ & $12(21.4)$ & 0.386 \\
\hline Streptococcus milleri group & $18(1.9)$ & $0(0.0)$ & $>0.999$ \\
\hline S. constellatus & 8 & 0 & \\
\hline S. intermedius & 11 & 0 & \\
\hline S. anginosus & 2 & 0 & \\
\hline Other viridans streptococci & $16(1.7)$ & $1(1.8)$ & 0.575 \\
\hline S. mitis/oralis & 10 & 0 & \\
\hline S. sanguis & 1 & 1 & \\
\hline S. gordonii & 3 & 0 & \\
\hline S. salivarius & 2 & 0 & \\
\hline Other streptococci species & $5(0.5)$ & $0(0.0)$ & $>0.999$ \\
\hline S. agalactiae & 2 & 0 & \\
\hline Not specified & 3 & 0 & \\
\hline Staphylococcus aureus & $49(5.1)$ & $2(3.6)$ & $>0.999$ \\
\hline Methicillin-susceptible & 34 & 2 & \\
\hline Methicillin-resistant & 15 & 0 & \\
\hline Klebsiella pneumoniae & $122(12.8)$ & $12(21.4)$ & 0.023 \\
\hline Pseudomonas aeruginosa & $32(3.4)$ & $0(0.0)$ & 0.402 \\
\hline Acinetobacter baumannii & $14(1.5)$ & $1(1.8)$ & 0.530 \\
\hline Enterococcus faecium & $4(0.4)$ & $0(0.0)$ & $>0.999$ \\
\hline Enterococcus faecalis & $2(0.2)$ & $0(0.0)$ & $>0.999$ \\
\hline Escherichia coli & $13(1.4)$ & $0(0.0)$ & $>0.999$ \\
\hline Stenotrophomonas maltophilia & $4(0.4)$ & $0(0.0)$ & $>0.999$ \\
\hline Hemophilus influenzae & $11(1.2)$ & $1(1.8)$ & 0.453 \\
\hline Serratia marcescens & $3(0.3)$ & $0(0.0)$ & $>0.999$ \\
\hline Proteus mirabilis & $2(0.2)$ & $0(0.0)$ & $>0.999$ \\
\hline Morganella morganii & $1(0.1)$ & $0(0.0)$ & $>0.999$ \\
\hline Mycoplasma pneumoniae & $152(15.9)$ & $9(16.1)$ & 0.665 \\
\hline Chlamydia pneumoniae & $250(26.2)$ & $14(25.0)$ & 0.735 \\
\hline Legionella pneumophila & $8(0.8)$ & $2(3.6)$ & 0.082 \\
\hline Virus & $65(6.8)$ & $2(3.6)$ & 0.770 \\
\hline Influenza A & 33 & 2 & \\
\hline Influenza B & 4 & 0 & \\
\hline Metapneumovirus & 7 & 0 & \\
\hline Rhinovirus & 10 & 0 & \\
\hline Adenovirus & 6 & 0 & \\
\hline Respiratory syncytial virus A & 2 & 0 & \\
\hline Respiratory syncytial virus B & 2 & 0 & \\
\hline Coronavirus OC43 & 2 & 0 & \\
\hline
\end{tabular}

Data are presented as $n(\%)$. In 842 patients, 1,010 pathogens were identified.

(47.9\%) in the negative CR group and 797/1,831 (43.5\%) in the control group. The distribution of pathogens was not significantly different between the negative CR and control groups, except for Klebsiella pneumoniae and L. pneumophila which were more commonly isolated in the negative CR group.

\section{CT Findings}

Of the 1,831 patients in the control group, 1,817 (99.2\%) had available CT scans. For patients hospitalized between 2015 and $2016(n=531)$, two radiologists (K.-M.S. and J.-K.L.) independently reviewed the CT findings and classified them into three groups (the kappa statistic was 0.943) [15]. For the remaining patients, one of the two radiologists determined CT findings. Compar- 
Table 5. Computed tomography findings of the patients

\begin{tabular}{|c|c|c|c|}
\hline & $\begin{array}{l}\text { Control } \\
(n=1817)\end{array}$ & $\begin{array}{l}\text { Negative CR } \\
(n=94)\end{array}$ & $p$ value \\
\hline \multicolumn{4}{|l|}{ Pulmonary parenchymal change } \\
\hline Consolidation & $1,720(94.7)$ & $72(76.6)$ & $<0.001$ \\
\hline GGO & $1,330(73.2)$ & $68(72.3)$ & 0.855 \\
\hline Centrilobular nodules or tree-in-bud & $475(26.1)$ & $39(41.5)$ & 0.001 \\
\hline \multicolumn{4}{|l|}{ CT pattern } \\
\hline Consolidation & $1,707(93.9)$ & $72(76.6)$ & $<0.001$ \\
\hline GGO-predominant & $43(2.4)$ & $8(8.5)$ & 0.003 \\
\hline Bronchiolitis-predominant & $33(1.8)$ & $14(14.9)$ & $<0.001$ \\
\hline No or minimal & $34(1.9)$ & $0(0)$ & 0.410 \\
\hline Necrotizing pneumonia on enhanced CT & $225 / 1,301(17.3)$ & $5 / 75(6.7)$ & 0.016 \\
\hline Pleural effusion & $752(41.4)$ & $11(11.7)$ & $<0.001$ \\
\hline Small & $516(28.4)$ & $10(10.6)$ & $<0.001$ \\
\hline Moderate & $147(8.1)$ & $1(1.1)$ & 0.013 \\
\hline Large & $89(4.9)$ & $0(0.0)$ & 0.020 \\
\hline Loculated pleural effusion & $210(11.6)$ & $2(2.1)$ & 0.005 \\
\hline
\end{tabular}

isons of CT findings between the two groups are presented in Table 5. Consolidation pattern was significantly less common (72 [76.6\%] vs. 1,707 [93.9\%], $p<0.001)$, and GGO-predominant (8 $[8.5 \%]$ vs. $43[2.4 \%], p=0.003)$ and bronchiolitis-predominant patterns (14 [14.9\%] vs. 33 $[1.8 \%], p<0.001)$ significantly more common in the negative $\mathrm{CR}$ group. The negative $\mathrm{CR}$ group had a lower frequency of necrotizing pneumonia (5/75 [6.7\%] vs. $225 / 1,301$ [17.3\%], $p=0.016)$ and pleural effusion (11 $[11.7 \%]$ vs. 752 [41.4\%], $p<0.001)$ than the control group.

\section{Discussion}

Negative CR findings in CAP patients who have positive CT findings may be attributed to the locations of the lesions, including the dependent lung, right middle lobe or lingula, and pattern of the lesions including GGO- or bronchiolitis-predominant pattern or small focal lesions. Clinically, CAP with negative CR findings was characterized by higher incidences of ECOG 4, bedridden status, and aspiration pneumonia; lower incidences of complicated parapneumonic effusion or empyema and pleural drainage; and lower blood levels of inflammatory markers. In terms of treatment outcome, LOS was shorter in the negative CR group than in the control group, while 30-day and in-hospital mortalities were similar between the two groups. The causative pathogens in the negative

Community-Acquired Pneumonia with

Negative Chest Radiograph
CR group were similar to those in the control group. On CT scan, the negative CR group exhibited higher frequencies of GGO- and bronchiolitis-predominant patterns and lower frequencies of consolidation, necrotizing pneumonia, and pleural effusion than the control group. As described in a previous study [16], at our institution, emergency physicians that encountered patients with suspected pneumonia attempted to confirm the diagnosis of pneumonia to transfer them from the Emergency Department to the Internal Medicine Department. For this reason, the majority of CAP patients in the present study underwent chest CT, although it is usually not necessary for the diagnosis of CAP.

In the present study, no abnormal findings suggestive of pneumonia were observed on CR in approximately $5 \%$ of hospitalized CAP patients, which was similar to the percentages (3-13\%) in previous studies $[1,4,7,12]$. The most common cause of negative CR findings in the present study was the location of the lesions in the dependent regions of the lungs, followed by GGO- or bronchiolitispredominant pattern on $\mathrm{CT}$ and presence of lesions in the right middle lobe or lingula. According to a previous study [7], the proposed reasons for pneumonic infiltration not being detected on CR images were as follows: (1) patchy, localized, or light opacity; (2) superimposition of the lesions on anatomic structures, such as the diaphragm and heart; and (3) lower accuracy of the anterior-posterior view compared to the posterior-anterior view. The di- 
aphragmatic dome and heart project over a significant portion of the anterior and basilar lung fields [22]. Pulmonary parenchymal changes in the dependent locations of the lungs may be superimposed on normal anatomic structures such as the diaphragm and heart, which results in the lesion being concealed, thereby leading to missed or delayed diagnosis of pneumonia. Bedridden patients cannot inspire fully due to comorbidities such as neurological disorders, and it is difficult to obtain a lateral radiographic view in such patients. In addition, in bedridden patients, the anterior-posterior view is used for CR imaging rather than the posterior-anterior view. Thus, a significant portion of the lung base is obscured in these patients [6]. In line with this, the negative CR group had higher frequencies of ECOG 4, bedridden status, and aspiration pneumonia. Therefore, in bedridden patients, chest CT may be valuable for the diagnosis of aspiration pneumonia. In the present study, in patients with a negative CR the anterior-posterior chest view was performed more frequently and the lateral view less frequently compared with the control group. Less accuracy of the anterior-posterior view, absence of the lateral view, and small size and subtle change of the lesions may also contribute to negative CR due to the lesions located in the dependent lung.

On CT, the negative CR group exhibited higher frequencies of GGO- and bronchiolitis-predominant patterns and lower frequencies of consolidation, necrotizing change, and pleural effusion. GGO- or bronchiolitis-predominant pattern is not likely to be easily detected on CR due to lighter density, although this pattern rarely occurs in CAP patients [15]. GGO and centrilobular nodules were observed on CT scans in elderly patients with acute lower respiratory infection and negative CR findings in a previous study [22]. Similar to another study [8], pneumonia lesions in the right middle lobe and lingula were also missed in our patients either because the lesions were small and focal, or because they were obscured by cardiac shadow.

The negative CR group exhibited lower blood levels of inflammatory markers and NT-proBNP, which was suggestive of a better prognosis. A previous study also suggested that CAP patients with negative CR and positive CT findings exhibited a lower blood procalcitonin level than those with positive CR findings [12]. We speculate that lower blood levels of inflammatory markers are associated with more localized and less dense lesions in CAP patients with negative CR findings. However, the proportions of patients with high CURB-65 scores and high PSI were similar between the negative CR and con- trol groups. Similarly, 30-day mortality, in-hospital mortality, and the rate of treatment success were not significantly different between the two groups. The negative CR group had significantly lower incidences of complicated parapneumonic effusion or empyema and pleural drainage than the control group. Previous studies have suggested that complicated parapneumonic effusion or empyema could prolong the LOS in hospitalized CAP patients $[23,24]$. This can partly explain the shorter LOS in the negative CR group in our study. In terms of treatment regimens used, initial antibiotic therapy was similar between the two groups, except for a lower frequency of antipseudomonal beta-lactam use in the negative CR group. The use of corticosteroids was less common in the negative CR group than in the control group. We speculate that higher levels of inflammatory markers and more extensive lesions in the control group may have affected the use of corticosteroids.

Several limitations of the present study should be noted. First, because the present study was retrospectively conducted in a single institution, the possibility of selection bias should be considered. However, the frequency of negative CR findings among CAP patients in our study was similar to that in a previous prospective multicenter study. Second, none of our patients underwent microbiological examinations for the presence of anaerobes in respiratory specimens. Hence, the possibility that anaerobes were not isolated as causative organisms cannot be ruled out. Moreover, due to the retrospective nature of this study, not all patients underwent the same tests for etiologic agents, including atypical pathogens. Furthermore, evaluation for respiratory viruses was performed in only some of the patients. Third, since this was a retrospective study, the selection of antibiotics was based on the judgment of the attending physicians. Fourth, the Charlson Comorbidity Index and the proportion of the hospitalized CAP patients with a CURB-65 score of 3-5 or a PSI class 4-5 were low in the present study. Medical expenses in South Korea are much lower than those in Western countries, and Koreans conventionally tend to be hospitalized and treated rather than to be treated in outpatient settings. Therefore, the proportion of hospitalized CAP patients was higher than expected in the present study. Finally, the number of patients in the negative CR group was too small to allow us to reach definitive conclusions.

In conclusion, negative CR findings in CAP patients in our study were due to the location of the lesions (dependent regions of the lung and the right middle lobe or lingula), CT patterns (GGO- or centrilobular nodule-pre- 
dominant), and small size of the lesions. Clinically, CAP patients with negative CR findings were characterized by lower blood levels of inflammatory markers, a higher incidence of aspiration pneumonia, and lower incidences of complicated parapneumonic effusion or empyema and pleural drainage. However, in terms of treatment outcome, no difference in short-term mortality was observed between the negative CR group and the control group, although LOS was shorter in the negative CR group. Chest CT scan should be considered in suspected CAP patients with a negative $\mathrm{CR}$, especially in bedridden patients, to diagnose pneumonia. In these patients, antibiotic treatment similar to that of CAP patients with a positive CR is needed.

\section{Disclosure Statement}

The authors declare no conflicts of interest associated with this study.

\section{References}

1 Claessens YE, Debray MP, Tubach F, Brun AL, Rammaert B, Hausfater P, et al. Early chest computed tomography scan to assist diagnosis and guide treatment decision for suspected community-acquired pneumonia. Am J Respir Crit Care Med. 2015 Oct;192(8):974-82.

2 Mandell LA, Wunderink RG, Anzueto A, Bartlett JG, Campbell GD, Dean NC, et al.; Infectious Diseases Society of America; American Thoracic Society. Infectious Diseases Society of America/American Thoracic Society consensus guidelines on the management of community-acquired pneumonia in adults. Clin Infect Dis. 2007 Mar;44 Suppl 2:S27-72.

3 Albaum MN, Hill LC, Murphy M, Li YH, Fuhrman CR, Britton CA, et al.; PORT Investigators. Interobserver reliability of the chest radiograph in community-acquired pneumonia. Chest. 1996 Aug;110(2):343-50.

4 Maughan BC, Asselin N, Carey JL, Sucov A, Valente JH. False-negative chest radiographs in emergency department diagnosis of pneumonia. R I Med J (2013). 2014 Aug 1;97(8): 20-3.

5 Self WH, Courtney DM, McNaughton CD, Wunderink RG, Kline JA. High discordance of chest $\mathrm{x}$-ray and computed tomography for detection of pulmonary opacities in ED patients: implications for diagnosing pneumonia. Am J Emerg Med. 2013 Feb;31(2):401-5.

6 Esayag Y, Nikitin I, Bar-Ziv J, Cytter R, Hadas-Halpern I, Zalut T, Yinnon AM. Diagnostic value of chest radiographs in bedridden patients suspected of having pneumonia. Am J Med. 2010 Jan;123(1):88.e1-5.

7 Haga T, Fukuoka M, Morita M, Cho K, Tatsumi K. Computed tomography for the diagnosis and evaluation of the severity of community-acquired pneumonia in the elderly. Intern Med. 2016;55(5):437-41.

8 Syrjälä H, Broas M, Suramo I, Ojala A, Lähde S. High-resolution computed tomography for the diagnosis of community-acquired pneumonia. Clin Infect Dis. 1998 Aug;27(2):35863.
9 Nambu A, Ozawa K, Kobayashi N, Tago M. Imaging of community-acquired pneumonia: roles of imaging examinations, imaging diagnosis of specific pathogens and discrimination from noninfectious diseases. World J Radiol. 2014 Oct; 6(10):779-93.

10 Sugishita K, Saito T, Asayama Y, Iwamoto T. Risk factors for detection failures of chest radiography in diagnosing pneumonia. J Gen Fam Med. 2017 Jun;18(6):398-402.

11 Hayden GE, Wrenn KW. Chest radiograph vs. computed tomography scan in the evaluation for pneumonia. J Emerg Med. 2009 Apr; 36(3):266-70.

12 Upchurch CP, Grijalva CG, Wunderink RG, Williams DJ, Waterer GW, Anderson EJ, et al. Community-acquired pneumonia visualized on CT scans but not chest radiographs: pathogens, severity, and clinical outcomes. Chest. 2018 Mar; 153(3):601-10.

13 Yeon Lee S, Cha SI, Seo H, Oh S, Choi KJ, Yoo SS, et al. Multimarker prognostication for hospitalized patients with community-acquired pneumonia. Intern Med. 2016;55(8): 887-93.

14 American Thoracic Society, Infectious Diseases Society of America. Guidelines for the management of adults with hospital-acquired, ventilator-associated, and healthcareassociated pneumonia. Am J Respir Crit Care Med. 2005 Feb;171(4):388-416.

15 Seo H, Cha SI, Shin KM, Lim JK, Yoo SS, Lee SY, et al. Relationship between clinical features and computed tomographic findings in hospitalized adult patients With communityacquired pneumonia. Am J Med Sci. 2018 Jul; 356(1):30-8.

16 Seo H, Cha SI, Shin KM, Lim JK, Yoo SS, Lee $J$, et al. Clinical relevance of necrotizing change in patients with community-acquired pneumonia. Respirology. 2017 Apr;22(3): $551-8$.
17 Fine MJ, Auble TE, Yealy DM, Hanusa BH, Weissfeld LA, Singer DE, et al. A prediction rule to identify low-risk patients with community-acquired pneumonia. N Engl J Med. 1997 Jan;336(4):243-50.

18 Lim WS, van der Eerden MM, Laing R, Boersma WG, Karalus N, Town GI, et al. Defining community acquired pneumonia severity on presentation to hospital: an international derivation and validation study. Thorax. 2003 May;58(5):377-82.

19 Oken MM, Creech RH, Tormey DC, Horton J, Davis TE, McFadden ET, et al. Toxicity and response criteria of the Eastern Cooperative Oncology Group. Am J Clin Oncol. 1982 Dec; 5(6):649-55.

20 Charlson ME, Pompei P, Ales KL, MacKenzie CR. A new method of classifying prognostic comorbidity in longitudinal studies: development and validation. J Chronic Dis. 1987; 40(5):373-83.

21 Hayashi M, Iwasaki T, Yamazaki Y, Takayasu $\mathrm{H}$, Tateno $\mathrm{H}$, Tazawa $\mathrm{S}$, et al. Clinical features and outcomes of aspiration pneumonia compared with non-aspiration pneumonia: a retrospective cohort study. J Infect Chemother. 2014 Jul;20(7):436-42.

22 Park JE, Kim Y, Lee SW, Shim SS, Lee JK, Lee $\mathrm{JH}$. The usefulness of low-dose CT scan in elderly patients with suspected acute lower respiratory infection in the emergency room. $\mathrm{Br}$ J Radiol. 2016;89(1060):20150654.

23 Kim J, Park JS, Cho YJ, Yoon HI, Lee JH, Lee $\mathrm{CT}$, et al. Predictors of prolonged stay in patients with community-acquired pneumonia and complicated parapneumonic effusion. Respirology. 2016 Jan;21(1):164-71.

24 Soriano T, Alegre J, Alemán C, Ruiz E, Vázquez A, Carrasco JL, et al. Factors influencing length of hospital stay in patients with bacterial pleural effusion. Respiration. 2005 Nov-Dec;72(6):587-93. 